\title{
Hepatic Fascioliasis, Unexpected Diagnosis and Atypical Presentation
}

\author{
Tarik Zaher ${ }^{1}$, Elsayed Saad ${ }^{1}$, Ahmad Elsamak ${ }^{2}$ \\ ${ }^{I}$ Tropical Medicine Department, Faculty of Medicine, Zagazig University, Egypt. \\ ${ }^{2}$ Radiology Department, Faculty of Medicine, Zagazig University, Egypt
}

Corresponding Author

Elsayed Saad Abd Elbaser (MD)

Mobile: 00201094986320

E mail: dr.sayedsaad79@ gmail.com

Key words: Hepatic ; Fascioliasis

\section{INTRODUCTION}

Hepatic Fascioliasis affects about 17 million people worldwide and outbreaks occur mostly in developing areas of the Caribbean [1]. In Egypt, the prevalence is variable. It is about $2-17 \%$ in different villages in Nile delta [2]. Humans are accidently infected after ingestion of encysted metacercaria contaminating watercress or water. Metacercaria are then developed into larva which penetrate duodenal wall to reach the liver. The disease has two phases; parenchymatous (acute/ hepatic) phase; characterized by fever, right upper quadrant pain, nausea, vomiting, jaundice, hepatomegaly and eosinophilia. The second phase is biliary (chronic), characterized by cholangitis and cholestasis [3].

Detection of Fasciola eggs in the stool is the most definitive test for diagnosis. Immunodiagnostic tests including enzyme immunoassays (EIA) and indirect haemagglutination assay (IHA) are useful in early phases of infection [4]. Imaging studies include abdominal ultrasonography and Computed tomography (CT) scanning may reveal characteristic lesions in the liver. Such findings may be confused with alternate diagnosis such as malignancy [5]. Abdominal ultrasonography findings in the parenchymal phase may include focal hypo-echoic or hyper-echoic lesions or diffuse involvement of the liver, while in the ductal phase there may be ductal dilatation which appears as hypo-echoic lines parallel to portal areas. Computed tomography (CT) findings in the parenchymal phase include multiple branching hypo-dense lesions, while in ductal phase there are dilated biliary ducts [6].

Here we present a case with hepatic focal lesions caused by Fasciola hepatica, misdiagnosed initially by her physician as hepatocellular carcinoma (HCC), due to its high incidence in our community, aiming at increasing the awareness of the physicians with such presentation.

\section{CASE REPORT :}

A 35 years old female living in a village belongs to Zagazig, a city in the Nile delta, Egypt, presented to another health institute with upper abdominal pain, anorexia for one month. Her blood results were as follow; normal complete blood count (CBC), increased alanine aminotransferase (ALT) and aspartate aminotransferase (AST) more than twice the upper limit of normal, alkaline phosphatase (ALP): $185 \mathrm{U} / \mathrm{L}$, gamma-glutamyl transpeptidase (GGT): $115 \mathrm{U} / \mathrm{L}$ and bilirubin: $1.5 \mathrm{mg} / \mathrm{dl}$. Abdominal ultrasonography showed hypo-echoic focal lesions with illdefined borders which were confirmed by abdominal CT. Her physician gave a probable diagnosis of HCC (based on high incidence of HCC in our community) and referred to our hospital. On admission the patient complained of upper abdominal pain. On examination there was no fever, no jaundice, and no organomegaly. Repeated laboratory tests showed normal CBC, ALT; 55 U/L, AST; 43U/L, ALP; 143 U/L, GGT; 96 U/L, bilirubin; $2 \mathrm{mg} / \mathrm{dl}$ and Alfa 
fetoprotein (AFP); $6 \mathrm{ng} / \mathrm{ml}$. Abdominal CT imaging confirmed the presence of multiple branching hypo-dense focal lesions in segments 7 and 8 of the liver but no cirrhosis (Fig. 1). Serology for viral hepatitis $(\mathrm{B}, \mathrm{C})$ was negative. However, repeated stool analysis revealed Fasciola eggs. Nitazoxanide was given for one month. Her symptoms resolved and liver biochemistry improved. However, CT abdomen showed regression of the lesions but didn't disappear completely, so, Triclabendazole $10 \mathrm{mg} / \mathrm{kg}$ was given once. Two months later, there was resolution of the focal lesions completely.

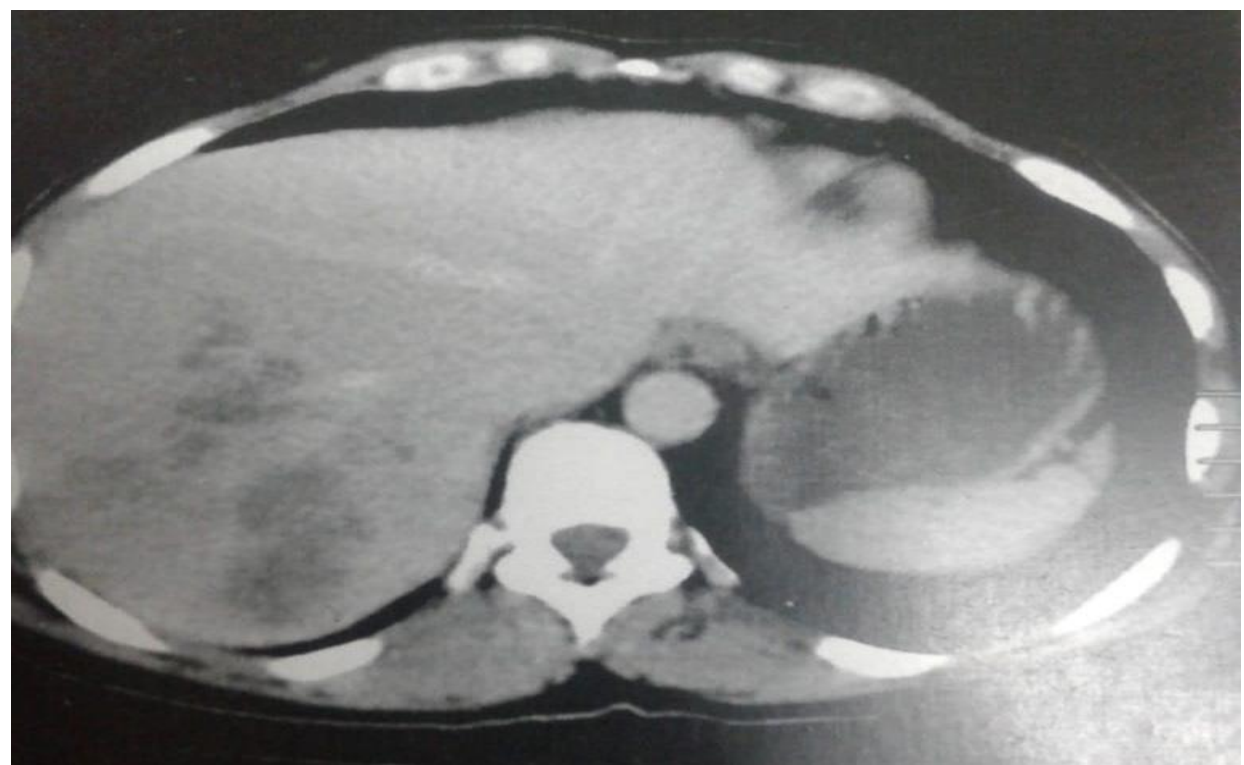

Figure (1) : Abdominal CT showed hypo-dense branching focal lesions in segments 7 and 8 in liver

\section{DISCUSSION}

Hepatic Fascioliasis is a neglected infectious disease despite its high prevalence in the developing countries. It is caused by the trematode Fasciola hepatica which is a parasite of herbivores (sheep, cattle) and human is accidentally infected. Human Fascioliasis has two important clinical phases; the hepatic or acute phase is characterized by fever, right upper quadrant pain, jaundice and eosinophilia, while the biliary or chronic phase is characterized by recurrent cholangitis or biliary obstruction [7]. Although our case presented with relevant clinical symptoms and signs, her laboratory tests didn't reveal eosinophilia. This may be attributed to high endemicity of the disease and acclimatization of the immune system to the parasite due to exposure through the whole life, or it may be due to immunosuppression, a state which wasn't encountered in our case.

Although detection of the fasciola eggs in the stool is the gold standard for the diagnosis [8], initial stool analysis of our case couldn't detect any ova. This may be attributed to presentation of the patient in the acute stage when immature worms feed on the liver tissue and no ova release. Also, absence of the ova in stool may be related to intermittent ova release [9]. Diagnosis of Fascioliasis should be confirmed by immunological tests including ELISA or HIA in case of absence of the eggs in stool.

Hepatic multiple focal lesions seen on routine abdominal ultrasonography is common in practice in our community due to high prevalence of hepatocellular carcinoma (HCC). However, the young age of the patient and absence of underlying liver disease together with the experience of our consultant with the radiological characteristics of fascioliasis, we made a suspicious of diagnosis. Further evaluation using abdominal computed tomography (CT) revealed characteristic branching hypo-dense lesions in segment 7 and 8 of the liver [10].

Triclabendazole (10-20 mg/kg/day) is the drug of choice for treatment of Fascioliasis [11]. Due to unavailability of this drug we used Nitazoxanide 
initially which gave some improvement. However, after treatment with Triclabendazole, complete resolution of the lesions was observed denoting its superiority over Nitazoxanide [12].

In conclusion, Fasciola hepatica should be kept in mind in the differential diagnosis of hepatic focal lesions, especially in younger patients with no history of chronic liver disease, since our country is endemic for it. We report this case to our physicians to be familiar and oriented with such atypical scenario.

Ethical consideration: Informed consent was taken from the patient.

Funding: None.

\section{Conflict of Interest : None.}

\section{REFERENCES}

1. Mas-Coma S, Valero MA, Bargues MD. Chapter 2. Fasciola, lymnaeids and human fascioliasis, with a global overview on disease transmission, epidemiology, evolutionary genetics, molecular epidemiology and control. Adv Parasitol, 2009; 69: 41-146.

2. Mas-coma S, Bargues M, Valero M. fasciolasis and otherplant-borne trematode zoonosis. Int $J$ Parasitol. 2005 oct. 35(11-12): 1255-78.

3. Harinasuta T, Pungpak S, Keystone JS. Trematode infections. Opisthorchiasis, clonorchiasis, fascioliasis, and paragonimiasis. Infect Dis Clin North Am, 1993; 7(3): 699-716.

4. Espinoza JR, Maco V, Marcos L, Saez S, Neyra $\mathrm{V}$, Terashima A et al. Evaluation of Fas2-ELISA for the serological detection of Fasciola hepatica infection in humans. Am J Trop Med Hyg, 2007; 76: $977-982$.
5. Lim JH, Mairiang E, Ahn GH. Biliary parasitic diseases including clonorchiasis, opisthorchiasis and fascioliasis. Abdom Imaging 2008; 33: 157165.

6. Dusak A, Onur MR, Cicek M, Firat U, Ren T, Dogra VS. Radiological Imaging Features of Fasciola hepatica Infection - A Pictorial Review. $J$ Clin Imaging Sci, 2012; 2:2.

7. Arjona R, Riancho JA, Aguado JM, Salesa R, González-Macías J. Fascioliasis in developed countries: a review of classic and aberrant forms of the disease. Medicine [Baltimore] 1995; 74: 13-23.

8. Valero MA, Perez-Crespo I, Periago MV, Khoubbane M, Mas-Coma S. Fluke egg characteristics for the diagnosis of human and animal fascioliasis by Fasciola hepatica and F. gigantica. Acta Trop, 2009; 111: 150-159.

9. Marcos LA, Terashima A, Gotuzzo E. Update on hepatobiliary flukes: fascioliasis, opisthorchiasis and clonorchiasis. Curr Opin Infect Dis 2008; 21: 523-530

10. Ince V, Ara C, Koç C, Ersan V, Barut B. Fasciola hepatica mimicking malignancy of the liver and colon: Three case reports. J Turgut Ozal Med Cent. 2010; 17: 207-10.

11. Lopez VR, Dominguez CA, Garron C. Successful treatment of human Fascioliasis with Triclabendazole. Eur J Clin Microbiol Infect Dis 1999; 18: 525-6.

12. Marcos LA, Tagle M, Terashima A, Bussalleu A, Ramirez C, Carrasco C et al. Natural history, clinicoradiologic correlates, and response to Triclabendazole in acute massive fascioliasis. $\mathrm{Am}$ J Trop Med Hyg 2008; 78: 222-7. 